\title{
L’Espace et la mer dans les contes bedjas (Soudan)
}

\author{
Martine Vanhove \\ (LLACAN, UMR 8135, INALCO-CNRS)
}

\section{Résumé}

Les contes bedjas du Soudan distinguent quatre espaces, terrestre, céleste, souterrain et maritime, qui sont très peu spécifiés (absence de toponymes ou repères directionnels, par exemple), mais seulement symbolisés par quelques éléments types de la société et de son environnement : huttes, puits, marchés, pâturages, arbres, tanières, grottes et oueds. Même les trajectoires des nombreux déplacements et voyages ne sont pas matérialisés dans des chemins ou sentiers. Après une étude des moments et des raisons des déplacements vers les espaces non domestiques, cet article s'attache plus particulièrement à l'analyse de l'espace maritime. Seule à ne posséder aucune caractéristique d'un territoire humain, la mer symbolise en effet par excellence le lieu de tous les dangers, dans une communauté qui lui tourne le dos alors qu'elle vit sur ses bordures.

MoTS CLES : bedja, contes, espace, voyages, mer.

\begin{abstract}
Four different spaces are distinguished in the Beja tales of the Sudan: earth, sky, underworld, and sea. All four are underspecified (e.g. no toponyms, no directional landmarks), and only alluded to with generic and symbolic items of the society and its ecology, e.g., huts, wells, markets, pastures, trees, grottos, and wadis. The trajectories of the numerous travels are not materialized by paths or trails. The first part of this paper investigates the timing and reasons of movements towards non-domestic areas, the second part focuses on an analysis of the maritime space. It is shown that the sea lacks any characteristics of the human territories and is the place of all possible dangers, a perception in line with the life style of the Beja people: although living in the vicinity of the Red Sea, they are traditionally neither fishermen nore sailors.
\end{abstract}

KEYWORDS: Beja, tales, space, travels, sea.

\section{Introduction}

La société bedja du Soudan ne valorise pas le genre du conte qui est qualifié, au même titre que la poésie chantée, de harar firid "parole vide». Il n'est d'ailleurs sans doute pas anodin que la dénomination même du conte n'appartienne pas à la terminologie autochtone, mais soit 
fondée sur des termes empruntés à l'arabe ${ }^{1}$ : gissa " histoire » et, plus souvent, massal ou mitaal ${ }^{2}$, le correspondant arabe ayant pour sens à la fois " histoire », " allégorie », " proverbe » et « exemple ». L'emprunt bedja présente une partie de cette polysémie puisqu'il signifie également " proverbe ». Quant à gissa, il désigne aussi les «anecdotes", notamment humoristiques.

La langue bedja ne distinguant pas ces différents genres littéraires, ce sont des critères structurels qui fondent une distinction que la culture effectue cependant. Les travaux de D. Morin (1995 : chap. 5) ont montré que le conte possède en propre des schèmes rythmiques caractéristiques et définitoires de ce genre.

Une autre spécificité, non exclusive du conte, concerne l'espace de la performance. Il relève de la sphère privée et la récitation publique par un professionnel n'existe pas. Les contes se disent à la maison, le soir à la veillée. Typiquement, l'énonciateur est une grand-mère et l'auditoire ses petits enfants, assis en demi-cercle autour d'elle. Il arrive aussi, mais de moins en moins, que, dans l'espace commun à l'extérieur de la hutte, les enfants ponctuent leurs jeux nocturnes collectifs par des contes, notamment pendant les longues soirées de Ramadan. Par ailleurs, contrairement à la zone $a \mathrm{fr}^{3}$, l'auditoire ne participe pas à la récitation du conte par des formules stéréotypées.

La très grande majorité du corpus ${ }^{4}$ qui a servi de base à cette étude a été enregistrée dans le cadre usuel de cette société, c'est-à-dire en famille, le soir, à la veillée. Si la mise en espace fait donc encore bien partie du conte, quelques contes ont cependant pu être enregistrés en contexte provoqué au cours de l'après-midi (contrairement à d'autres sociétés, les Bedjas ne semblent pas avoir d'interdiction formelle concernant la période propice à la récitation des contes).

1 Voir M.-T. Hamid Ahmed (2005) sur le rôle sociolinguistique de l'arabe, notamment son utilisation pour éviter de transgresser les règles éthiques de la communauté bedja.

2 Il s’agit de variantes dialectales, la première étant utilisée au sud, la seconde au nord de l'aire linguistique bedja.

3 Autre langue couchitique parlée en Erythrée, Ethiopie et Djibouti.

4 Les contes étudiés sont ceux que j’ai recueillis sur le terrain entre 2000 et 2003 au cours de quatre missions. Ce corpus est en cours de constitution et ne comporte pas encore de variantes; il se compose actuellement d'un total de quarante-trois contes, de durées différentes. La majeure partie des contes a été recueillie auprès d'un jeune homme de Sinkat, âgé d’une trentaine d'années, et qui les avaient mémorisés dans sa jeunesse en écoutant les récits d'une vieille voisine. Il a conservé, pour un homme adulte, un goût inhabituel et prononcé pour ce genre littéraire. Quelques autres contes m’ont été racontés par ses frères, sœurs et mère, deux autres hommes et quelques enfants de Wagar. 
Après une caractérisation générale de l'espace dans le conte bedja, cette présentation se propose d'étudier plus particulièrement le rôle que joue la mer dans une culture qui la rejette alors qu'elle vit sur ses bordures.

\section{L'espace dans les contes}

Fondamentalement, l'espace dans les contes se divise en quatre entités physiques en référence à une bi-partition humain - non humain.

Le monde des humains (ou éventuellement de leurs homologues animaux) est celui de la réalité. Il est présent dans tous les contes, mais n’est jamais désigné comme tel. Il se déduit des récits par un certain nombre d'indices, notamment spatiaux. C'est un espace terrestre comprenant deux parties : le territoire de soi-même ou, par extension, de la communauté (daamar, haaš), en général représenté par la maison (gaw) ou la famille, le foyer (diwa), et l'espace lointain, à savoir la brousse ou le territoire des autres (matlal).

Si le terme haaš est relativement fréquent dans les contes, par contre celui de daamar est rare. Il n'est apparu que dans deux contes à caractère merveilleux. Quant au terme matlal, il n'est jamais utilisé dans le corpus, mais est le plus souvent évoqué par d'autres termes : soit par un syntagme à connotation sémantique vague, le pays lointain (saagi balaad), soit par des termes où l'espace d'autrui est dénoté plus spécifiquement : la forêt dense (daw), la forêt (gaaba), le lieu désertique, l'espace inhabité (ma/ingaay), le désert (sabboot), la montagne (riba). Les contes peuvent se dérouler dans l'un ou l'autre espace et les personnages effectuent de fréquents passages de l'un à l'autre.

Le monde des non humains comporte :

(i) des monstres : êtres fabuleux souvent reptiliens, ils n'appartiennent pas à la vie quotidienne,

(ii) des djinns: très présents dans l'univers quotidien, ils sont considérés comme réels et empruntent des apparences diverses, animale (domestique ou sauvage) ou humaine, qui leur permettent de se fondre dans le monde des humains. Seul leur comportement les trahit et les désigne comme intrus.

Pas plus qu'il n'est question explicitement du «monde des humains », il n’est fait référence à un «monde non humain », mais seuls les non humains peuplent sans problème quatre espaces différents :

- Le plus souvent, les djinns se meuvent, comme les hommes, dans l'espace terrestre, qu'il s'agisse du territoire restreint de la famille ou de celui plus large de la communauté (haaš, daamar), ou, moins fréquemment, du territoire des autres, parfois précisé comme étant la 
brousse, le désert ou la montagne. Plus rarement, les monstres peuvent également y pénétrer, mais seulement pour y commettre des méfaits qui vont engendrer une quête.

- L'espace céleste est peu présent dans mon corpus: dans un seul conte, le ciel (too-biri) est mentionné comme le lieu de résidence d'un chien djinn. Il s’y est réfugié après avoir capturé, sur terre, la femme de son maître. La femme y est retenue prisonnière, mais l'homme n'y pénètre pas au cours de sa quête ; ce sont les animaux qui lui servent d'intermédiaires. Cet espace céleste est l'exact reflet de l'espace terrestre : on y trouve, par exemple, des arbres et des maisons.

Quant aux oiseaux, créatures qui vivent dans les airs, ils sont le plus souvent des auxiliaires magiques bienfaiteurs qui, par exemple, permettent à l'homme de voler pour se sortir d'une mauvaise passe. Les oiseaux monstrueux sont rares et il s'agit toujours d'oiseaux marins.

- Djinns et monstres, quoique rarement, peuvent partager l'espace souterrain. Seuls deux contes s'y déroulent en partie. L'accès à cet espace, dans lequel il arrive aux humains de descendre en personne pour y subir des épreuves liées à leur quête, s'effectue soit par un puits, soit par une grotte. Il possède toutes les caractéristiques du monde terrestre.

- Enfin, l'espace maritime, plus présent dans les contes que les deux précédents, est lui aussi peuplé de monstres et de djinns. La mer (bhar, un emprunt à l'arabe) est ainsi la garantie de l'immortalité du chien djinn qui $\mathrm{y}$ abrite son cœur. C'est le seul espace qui ne ressemble en rien à l'espace terrestre.

Les contes présentent donc un espace pluriel à quatre composantes, dont l'une est privilégiée, l'espace terrestre, et où seul l'homme est implanté de manière permanente et légitime. Mais c'est un espace ouvert auquel les djinns, et plus rarement les monstres, ont accès sans difficulté. A l'inverse, s’il arrive aux humains de pénétrer dans les espaces non terrestres, c'est toujours pour y subir des épreuves qui ponctuent leurs quêtes. L'homme n'est véritablement à sa place que sur terre et plus spécifiquement dans les contes, sur le territoire de la communauté, dans l'espace domestique, même si c'est souvent au cours de sa quête dans les espaces terrestres lointains d'autrui, symboles de liberté ( $c f$. M.-T. Hamid Ahmed dans ce volume), qu'il gagne sa légitimité d'être humain (en épousant la fille d'un chef, par exemple).

Il convient de souligner dès à présent que la mer, pourtant facilement accessible à l'homme dans la réalité, comme dans les contes (elle s'atteint sans obstacles tels que puits, grottes ou incapacité de voler), n'est pas considérée comme un espace approprié pour les humains. Si l'espace maritime est peuplé de non humains et si l'homme n’y pénètre jamais 
dans son vécu quotidien, il se situe de surcroît très nettement en marge puisqu'il ne rappelle en rien, par sa topographie, les territoires humains.

Outre les références aux quatre espaces dont il vient d'être question, les contes présentent également quelques indications géographiques récurrentes à caractère social ou naturel. Dans la société pastorale bedja, qui ne pratique l'agriculture de subsistance que de manière marginale, le champ n'est que rarement l'objet d'un épisode du conte, en l'occurrence un méfait engendrant une quête. Par contre les pâturages, symbole par excellence de l'activité essentielle des Bedjas, le puits et le marché, lieux sociaux de rassemblement des hommes (exclusivement), sont souvent des éléments constitutifs importants où se nouent les épreuves que devra surmonter le héros ou les péripéties du conte. Dans l'espace naturel de cette région aride, qu'il soit domestique ou sauvage, l'élément végétal symbolisé par l'arbre joue un rôle de premier plan, notamment l'acacia ( sag? $\left.^{2} a n i\right)$, le palmier doum (šaašoot) et le jujubier (saroob), associés aux djinns et aux animaux sauvages dangereux. S'y ajoutent des éléments minéraux, construits ou naturels, que sont la tanière, le terrier et la grotte, eux aussi liés aux djinns et aux animaux sauvages. Enfin, l'oued, dont les crues peuvent être redoutables, même s'il est rarement mentionné, n’a pas une fonction unique. Il peut être un simple repère topographique (le plus souvent dans les parties poétiques des contes), ou la cachette d'un animal monstrueux (rappelant ainsi la méfiance vis-à-vis de la mer) ou encore le support symbolique d'un acte de sagesse. Cette ambivalence de l'oued dans le conte est peut-être le reflet de son double caractère de bienfait pour les cultures et la vie et de dévastation et de mort lors des crues trop importantes. On ne peut pas exclure non plus l'effet d'une certaine forme d'acculturation liée à l'islamisation (fort ancienne) des Bedjas. Les deux hypothèses ne sont pas exclusives l'une de l'autre, mais seul un corpus plus vaste, confronté à un corpus de contes arabes soudanais et yéménites ${ }^{5}$, permettrait de prendre position de manière plus assurée.

Ce n'est guère que dans quelques rares contes, à l'évidence adaptés du répertoire arabe, qu'apparaissent des espaces sociaux urbains tels que le jardin, le palais, la mosquée, le tribunal ou la rue, qui, à l'exception de la mosquée, étaient encore assez récemment étrangers à la société bedja.

Le chemin, sous sa forme socialisée de rue ou plus naturelle de sentier, malgré les nombreux déplacements effectués par les protagonistes des contes, ne figure explicitement que dans deux contes du corpus, et

5 Les contacts sont anciens et persistants avec la côte yéménite et ses habitants, dont certains sont même installés dans l'est du Soudan. 
encore n'est-il pas un lieu de passage, mais un endroit où se déroulent des épisodes statiques (les nattes d'une tente s'y sont dispersées, par exemple).

Dans le conte bedja, le voyage, la transhumance et l'errance sont autant d'éléments constitutifs. Les personnages effectuent de nombreux déplacements dans l'espace terrestre, lointain ou domestique, mais aussi parfois, et malgré eux, dans les trois autres.

L'une des principales raisons qui poussent les héros humains au départ est la quête de nourriture, soit par la recherche d'un travail, soit par le vol, le plus souvent vers un pays lointain ou le territoire d'autrui. Ces quêtes sont ponctuées de nombreuses épreuves dans les espaces autres que terrestre et se soldent par un retour victorieux dans le foyer d'origine. Si les déplacements sont parfois courts, ils ont alors lieu entre l'espace domestique et la mer.

La quête d'une épouse est aussi une raison des déplacements. Ponctuée d'épreuves, elle est souvent initiée par la poursuite d'un monstre ou d'un djinn, ou parfois par la vue d'une belle femme. Plus rarement, c'est la femme qui initie involontairement la quête, soit en fuyant une activité désagréable, soit grâce à un don surnaturel qui conduit à des jalousies, des mésaventures et finalement à des épousailles. Cette quête entraîne toujours la traversée de pays lointains, du monde souterrain ou de la mer. L'installation finale du héros peut se faire soit dans le pays de son épouse, soit dans son foyer d'origine. Dans un seul cas, la quête se termine par le choix de la mauvaise épouse (une djinn) et la mort du héros de retour chez lui.

D'autres déplacements initiés par un monstre ou un djinn visent seulement à mettre un terme à leurs méfaits en les tuant, par exemple après être allé acheter une arme au marché.

Les voyages des héros peuvent aussi être contraints par d'autres humains : des voisins obligent une jeune fille à aller nager en mer et elle finit enlevée par un djinn dans un fleuve puis entraînée au ciel, tandis que son frère est transformé en chien ; une communauté chasse des idiots infanticides qui perdront leurs provisions et s'entretueront ; des voleurs fuient leurs poursuivants vers des territoires d'autrui pour y subir, près d'un puits ou d'un réservoir, une épreuve qui permettra leur intégration dans une nouvelle communauté ; une fille simplette fuit son frère trop sévère et revient riche au foyer grâce à sa bêtise ; une mère et un fils fuient un tyran et, grâce à une meule en or, le fils retourne au pays et devient vice-roi ; une marâtre jalouse fait exiler sa belle-fille au loin ou cherche à la noyer en mer. Il se peut aussi que la perte du bétail motive un départ. Toutes ces fuites, exils et quêtes ont pour destination les espaces 
non domestiques : la montagne, le désert, le territoire d'autrui, un pays lointain, la mer, les repères des djinns.

D’autres déplacements des héros sont motivés par des tâches domestiques : emmener le bétail aux pâturages, faire la lessive au bord de la mer, aller chercher de l'eau au puits. Ils entraînent une série d'épreuves qui se déroulent uniquement dans l'espace terrestre, qu'il s'agisse du territoire domestique ou de celui d'autrui. Ils aboutissent à un enrichissement et au retour au foyer.

Il est aussi des déplacements, assez rares, qui ne sont pas explicitement motivés dans le conte. Ainsi peut-il être simplement fait allusion au fait qu'un frère et une sœur se rendent au bord d'un fleuve sans que la raison en soit précisée. La fille y est enlevée par un lion et c'est grâce à l'intervention d'une colombe que son frère la délivrera.

Dans un seul cas, c'est une intervention divine qui, en dupliquant une fille unique pour honorer les promesses de mariage inconsidérées de son père à plusieurs prétendants, entraînera le départ de celui-ci pour retrouver sa véritable fille.

De manière générale le conte commence donc par le départ d'un lieu, mais il arrive parfois que ce soit l'arrivée en un lieu qui déclenche le récit. C’est alors toujours un protagoniste secondaire qui pénètre dans le territoire du héros principal, soit pour finalement en être chassé, soit pour en chasser ce dernier.

Tous ces déplacements se caractérisent par une absence quasi totale de toponymes et de repères directionnels explicites ou implicites. Les très rares exceptions se situent dans les parties poétiques des contes, pour lesquelles l'espace est justement définitoire ( $c f$. l'article de M.-T. Hamid Ahmed dans ce volume), et dans des versions incluant des éléments du monde moderne ${ }^{6}$ (par exemple Renarde qui part en voiture voler dans la grande ville de Kassala).

\section{La mer dans le conte bedja}

Parmi ces quatre espaces principaux que sont les espaces terrestre, souterrain, céleste et maritime, il arrive que les humains soient obligés, dans leur quête ou leurs mésaventures, de pénétrer ou de côtoyer un des espaces propres aux djinns et aux monstres, en l'occurrence la mer. Il convient de rappeler à ce propos que, traditionnellement, les Bedjas, qui pourtant vivent le long de la mer Rouge, transhumant entre la plaine

6 Il existe également, dans les contes hagiographiques issus de la tradition musulmane et dont il ne sera pas question ici, une opposition entre le monde divin, dont l'emplacement et les caractéristiques sont totalement indéterminés, et le bas monde (dinya) bien terrestre. 
côtière et les montagnes qui la bordent, ne sont pas tournés vers la mer : ils ne consomment pas de poisson, à l'exception des quelques rares clans méprisés de pêcheurs, et ne sont pas marins. Cet aspect de la culture bedja, vraisemblablement un héritage historique lié aux déplacements forcés des populations, se manifeste dans le conte. Pour cette société, la mer, proche dans l'environnement, mais éloignée culturellement, est une figure inquiétante dont on se méfie généralement. Le conte explicite à sa façon cette méfiance.

Dans le corpus étudié ici, l'espace maritime apparaît dans onze des quarante-trois contes. $\mathrm{Si}$, au simple plan quantitatif, la mer est un élément spatial relativement marginal en comparaison de l'espace terrestre toujours présent, il faut rappeler qu'elle l'est toutefois beaucoup moins que les espaces souterrain (deux contes) et céleste (un conte). Elle occupe donc bien une place privilégiée, voire centrale, parmi les espaces non terrestres et non humains, place qui, entre autres, justifie l'attention plus grande qui y est portée ici.

Alors que la mer est peuplée de monstres et de djinns et constitue un de leurs domaines propres, ils ne sont qu'assez rarement mentionnés dans les onze contes, puisqu'il n’y est fait référence que trois fois.

Dans le premier cas, le plus anodin et le plus neutre, la mer et les monstres qui la peuplent sont offerts au héros dans un bidon ${ }^{7}$ comme l'un des trois auxiliaires magiques pour confectionner les robes qui lui permettront de surmonter une dernière épreuve et d'aboutir dans sa quête de la bonne épouse. La mer n'est certes pas dans ce conte un espace où se meuvent les personnages et n'a pas de fonction négative, mais il est important de noter que les monstres sont donnés par le conteur comme une de ses parties constitutives, au même titre que les poissons, ou que, pour le ciel, les étoiles et la lune. Ce conte n'est pas sans rappeler l'ambivalence de l'oued mentionné plus haut et il n'est pas impossible qu'il soit une adaptation du répertoire arabe (ce qui demande toutefois à être vérifié).

Le deuxième cas est celui du conte du chien djinn dont il a été question plus haut et dans lequel la mer est le lieu maléfique où le djinn abrite son cœur pour se protéger de la mort. Le héros, qui n’y pénètre pas directement, réussira à en prendre possession grâce au crocodile, l'un des quatre auxiliaires magiques que sont les animaux de ce conte. Il pourra ainsi faire libérer sa femme capturée par le djinn. La mer n'est donc pas directement à l'origine d'un méfait ou d'une mésaventure; elle est

7 Deux autres bidons contiennent « la terre avec ses hyènes, ses gazelles et ses arbres » et « le ciel avec ses étoiles et sa lune ». 
néanmoins la gardienne de l'immortalité du djinn par qui le malheur est arrivé.

Dans le troisième conte, la mer et ses monstres sont vraiment à l'origine d'une série de mésaventures. Au début du conte, le héros part chercher du travail, en trouve sur un bateau qui, une fois en mer, est attaqué par un monstre marin. Le capitaine et ses employés s'en débarrassent en passant par dessus bord toute leur cargaison de bétail et de nourriture. Puis un oiseau marin survient, les capture un à un pour les donner à manger à ses petits. Le héros et le capitaine en réchappent, mais leur traversée maritime suivante, sans intervention d'un monstre quelconque cette fois, se soldera par leur capture comme esclaves, une fois arrivés au port, lieu du marché aux esclaves.

Dans la plupart des contes du corpus de référence, la fonction négative de la mer n'est donc pas directement liée à sa population. Elle se manifeste dans différentes épreuves, méfaits et mésaventures, comme celle qui vient d'être évoquée avec le marché aux esclaves.

Sur le plan de la structure du récit, il est à noter que les méfaits et épreuves dont l'espace maritime est la cause ou le lieu de déroulement ne sont pas propres à un seul moment du conte. La mer peut apparaître aussi bien au début, au milieu, qu'à la fin des contes, dans cet ordre pour ce qui est de la fréquence.

Dans les cinq contes où la mer est un constituant spatial initial, celleci est soit la cause directe ou indirecte des mésaventures et des épreuves que vont subir les héros, soit le lieu où ils se déroulent. En dehors du conte à plusieurs séquences avec le monstre marin dont il vient d'être question, le corpus présente quatre autres situations. Dans les deux premières, la mer est pourvoyeuse d'une nourriture, méprisée par la société (les poissons et les mouettes), qui vient à se tarir suite à une transgression d'un des personnages des contes: (i) un pêcheur voit sa pêche diminuer quand sa femme tue un de leurs enfants, car elle pensait ainsi pouvoir mieux nourrir son époux ; (ii) une femme n'arrive plus à chasser de mouettes au bord de la mer pour nourrir ses enfants quand son époux se mêle d'en faire autant sans se cacher, entraînant la capture de toute la famille par une sorcière. La morale semble bien être : qui touche à la mer, de près ou de loin, est puni par la mer. Dans les deux autres situations, la mer déclenche la dégradation des conditions de vie des protagonistes : (iii) une jeune fille simplette se fait happer sa lessive par la mer et ce sera le début de l'appauvrissement de son frère ; (iv) une autre jeune fille, obligée de nager dans la mer, perd le collier que ses voisines lui ont offert, ce qui conduira à sa fin tragique et à celle de son 
frère après qu'un monstre, serpent d'eau douce à sept têtes, le lui aura repêché.

Dans quatre contes du corpus, c'est au milieu du récit que les personnages se rendent en mer ou sur son rivage. Il convient d'emblée d'écarter l'un d'eux, celui déjà mentionné où la mer n'est qu'un auxiliaire magique. Peut-être faut-il aussi regrouper un autre conte avec les précédents dont le début se situe dans l'espace maritime. Il s'agit en effet d'un long conte à plusieurs séquences dont la première est une variante d'un motif connu par ailleurs dans le domaine arabe au Soudan sur le choix de l'ami sincère ( $c f$. V. A. Yagi, 1981). Dans la deuxième séquence, les deux amis commencent par se rendre au bord de la mer où vogue un bateau. L'un d'eux tombe amoureux de sa passagère et en devient malade. C'est le point de départ de nombreuses mésaventures et d'une longue quête semée d'embûches pour la retrouver et finalement l'épouser.

Dans les deux autres contes, la mer a deux fonctions négatives différentes. Dans un cas, c'est le lieu de l'accomplissement d'un méfait au cours duquel l'héroïne subit une épreuve : durant le voyage qu'elle effectue avec sa marâtre (qui la déteste) pour aller épouser un prince, cette dernière lui arrache les yeux, l'enferme dans une malle et la jette à la mer. Dans l'autre conte, la mer constitue un obstacle que doit franchir, en le sautant à cheval et en récitant un poème, le héros poursuivi par les sœurs de sa belle qu'il a enlevée.

Quant à l'unique conte où la mer est le lieu de destination finale d'une des protagonistes, il me semble emblématique de ce rejet viscéral de la mer, de cette vision négative qui caractérise la culture bedja. C'est en effet le lieu de refuge, mais aussi de bannissement de la société, d'une jeune fille qui a transgressé deux tabous en aguichant un homme handicapé et en refusant de l'épouser.

\section{Conclusion}

Dans le conte bedja, la mer est donc bien un obstacle et le lieu de tous les dangers pour les humains. Peu importe d'ailleurs leur position par rapport à celle-ci, qu'ils soient dedans ou au bord, il suffit de l'approcher pour que le danger se manifeste à coup sûr. S'il est souvent possible de surmonter l'obstacle et les épreuves par la ruse ou des auxiliaires magiques, il faut souligner que c'est le seul espace dont l'homme ne sort pas toujours victorieux lors de son affrontement avec lui, comme le montrent la fin tragique d'un conte où l'héroïne et son frère trouvent la mort et le bannissement de la jeune fille qui a transgressé deux tabous. Le conte bedja dit bien qu'il ne fait pas bon s'aventurer en mer, ni même s'en approcher ! Il exprime très nettement que celle-ci n'est pas un espace 
humain, certes au même titre que le ciel ou l'espace souterrain, mais qu'elle est d'autant plus dangereuse qu'elle est une réalité qui s'impose à l'homme. La repousser aux frontières des espaces, en la privant de toute caractéristique terrestre dans sa constitution est aussi une manière de la nier.

Références bibliographiques

HAMID AHMED, M.-T., 2005, Ethics and Oral Poetry in Beja Society (Sudan), in Miller, Catherine (ed.) with collaboration of F. Ireton \& I. Dalmau, Land, ethnicity and political legitimacy in Eastern Sudan, Kassala \& Gedaref States, Cairo, Cedej, pp. 473-502.

MORIN, D., 1995, "Des paroles douces comme la soie". Introduction aux contes dans l'aire couchitique (bedja, afar, saho, somali), Paris, Peeters, $384 \mathrm{p}$.

YAGI, V. A., 1981, Contes d'Omdurman, Antibes, Bibliothèque Peiresc - Aresae, 287 p. 